\title{
EL LOGRO OCUPACIONAL EN ESPAÑA, VEINTE AÑOS DESPUES
}

\section{Rafael Gobernado Arribas}

Universidad de Málaga

En 1975, la OCDE publicó un conjunto de informes cuyo objetivo común era el análisis del papel que desempeñaba la educación frente a la desigualdad social en diferentes países. Entre esos informes hay uno dedicado a la situación correspondiente en España, redactado por J. Díez Nicolás, U. Martínez Lázaro y J. M. Porro Minondo (1975). La particularidad de este informe, para nosotros, se debe a que incorpora el primer análisis path de la estructura social española, siguiendo los pasos del status attainment (logro ocupacional) iniciado por O. D. Duncan y P. Blau (1967) en los años sesenta en Norteamérica (véanse L. Cachón Rodríguez, 1989: 301-315; D. W. Rossides, 1990: 102-104 y 174-175). Hoy, veinte años después, vale la pena intentar comparar aquellos resultados con los obtenidos en un nuevo análisis path de la sociedad española de mediados los años noventa.

Frente al redondeo de las fechas anteriores, la realidad impone su irregularidad: el trabajo de J. Díez Nicolás y colaboradores se basa fundamentalmente en datos secundarios obtenidos de una encuesta realizada en 1965 para el entonces existente Instituto de la Opinión Pública. Por su parte, nuestra investigación utiliza datos secundarios de 1993, en este caso provenientes del CIRES. Así, pues, más que veinte años después, debería decirse veintiocho años después.

Desde 1975 hasta nuestros días, han sido escasos los trabajos en España que han aplicado esta técnica cuantitativa de análisis y, en general, con un alcance local (J. Carabaña, 1983, con una muestra de población sobre Madrid; 
F. Requena, 1991, con una muestra sobre la ciudad de Málaga). Sin embargo, en 1993 se publica un amplio e importante trabajo sobre el logro ocupacional en España, elaborado por J. Rodríguez Menés (1993) a partir de una macroencuesta $(\mathrm{N}=27.377)$ realizada en 1988 por el Centro de Investigaciones Sociológicas. El objetivo de Rodríguez Menés se centra en el momento de entrada al mercado laboral del entrevistado, más concretamente se centra en la primera ocupación del entrevistado, lo que le separa del modelo de J. Díez Nicolás y, por lo tanto, de nuestros objetivos. De cualquier forma, los resultados de ese estudio sirven como orientación en nuestro trabajo.

La principal razón de que en España no hayan menudeado más estudios de análisis del logro ocupacional se debe seguramente a la ausencia en nuestro país de una escala ocupacional basada en el prestigio, convenientemente elaborada e institucionalizada dentro de las ciencias sociales.

\section{MARCO INTERPRETATIVO}

A. Los problemas que plantea una comparación de este tipo son múltiples. Surge la primera dificultad al tener que decidir si el modelo utilizado de análisis path, tanto el de 1975 como el de 1995, es ya de por sí explicativo (tiene base teórica) o si, por el contrario, es preciso utilizar otro modelo previo de cambio social que dé cuenta de los datos. Este problema no es nuevo y ha sido sacado a la luz recientemente en nuestro país (T. Hernández de Frutos, 1993). En efecto, son muchos los autores que no reconocen categoría teórica a los estudios sobre el logro ocupacional (especialmente demoledor al respecto fue L. A. Coser, 1975). Esta opinión obligaría a interpretar los resultados empíricos obtenidos según modelos teóricos ajenos a tal logro ocupacional.

Sin embargo, las técnicas de análisis de datos no están libres de connotaciones teóricas, y la que estamos barajando todavía menos. En el fondo de los trabajos sobre el «logro ocupacional» subyace la idea de modernización de Weber: la racionalidad, la eficacia y la individuación como procesos que paulatinamente se van implantando en la sociedad según se desarrolla técnica y económicamente (J. D. Knottnerus, 1987).

La clave de este tipo de análisis es la individuación. En efecto, el análisis path es diferente, por ejemplo, del análisis de movilidad social (merece destacarse el equívoco que supone el título del trabajo de J. Rodríguez Menés ya citado, que habla de "movilidad social» donde debería hablarse de "logro ocupacional»). La movilidad social tiene por objeto de estudio a sociedades, agrupaciones. Es un instrumento que sirve para determinar el grado de permeabilidad existente entre las categorías sociales que conforman las sociedades. El análisis path, por su parte, estudia las causas que influyen en la variación de los itinerarios individuales. Dirige la atención a los sucesivos estadios de la vida de los individuos (R. Girod, 1977). Encaja, por lo tanto, en los modelos teóricos de medios-fines; de estructura y acción. En otras palabras, el análisis path tiene 
validez para revalidar hipótesis propias del logro ocupacional (el actor, mediante el esfuerzo y aprovechamiento educativo, puede alterar la trayectoria reproductora de la sociedad). Por el contrario, y a título de ejemplo, apenas sería útil para constatar hipótesis provenientes de la teoría neomarxista de la reproducción social (P. Bourdieu, 1981), constatación que sí sería posible con las tablas de movilidad social.

En resumen, el análisis path da cuenta de en qué medida una sociedad posibilita más la adquisición que la adscripción de los roles. Más concretamente, informa sobre qué tipo de estrategia es la preferida por los individuos o sus familias: la adscriptiva o la adquisitiva. Si tenemos en cuenta el papel que juega la educación en el proceso de adquisición, es más completo recordar entonces que el «logro ocupacional» mide el nivel de meritocracia de una sociedad. El análisis en cuestión incorpora, por lo tanto, un enfoque teórico sustantivo, pese a la idea de Coser.

B. Las estructuras posibilitan el comportamiento de los actores, tanto positiva como negativamente. Ahora bien, sabemos que las estructuras sociales cambian en gran medida a causa precisamente de ese constante intento de logro profesional de sus miembros. La comparación que nos ocupa, entre el modelo de logro ocupacional de 1975 y el de 1995, debe considerar estas variaciones. Precisamente tales variaciones constituyen la parte explicativa que no incorpora el modelo estudiado. El cambio social que se detecta entre ambas fechas ha de interpretarse, por lo tanto, con información empírica y lógicodeductiva que va más allá de los propios modelos de logro ocupacional.

Los cambios habidos en las variables consideradas en el modelo pueden resumirse en los siguientes:

1. Existe una mejora (de renta y de prestigio) global de la población ocupada en la actualidad.

2. ${ }^{\circ}$ No obstante, se mantiene parecido nivel de desigualdad social, pese a la mejora global.

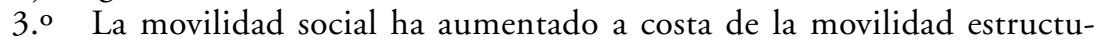
ral, manteniéndose semejante la movilidad de circulación.

4. ${ }^{\circ}$ La estructura educativa sufre un proceso de igualación social hacia arriba (disminuyen los niveles educativos inferiores y aumentan los superiores).

5. ${ }^{\circ}$ La evolución de la estructura ocupacional difiere, por lo tanto, de la evolución de la estructura educativa (L. C. Thurow, 1977).

6. La expansión educativa en sí misma justifica la independencia entre el nivel educativo y el origen social.

7. ${ }^{\circ}$ La expansión educativa a la larga independiza el nivel educativo de la clase ocupacional de pertenencia y de la renta.

8. ${ }^{\circ}$ La independencia entre educación formal y ocupación hace aumentar la importancia del origen social en el logro ocupacional (R. Boudon, 1983: 155-197). 
C. El entorno institucional del sistema educativo cobra una importancia extraordinaria en este tipo de análisis. Cualquier variación en el mismo puede desencadenar rupturas evidentes en las posibles tendencias del logro ocupacional. Así lo han entendido diversos autores que han utilizado el análisis path para determinar de forma empírica el alcance de modificaciones institucionales de los respectivos sistemas educativos (A. H. Halsey, 1977; D. H. Krymkowski, 1991).

En nuestro caso, la ruptura institucional más evidente se debió a la implantación de la reforma educativa de Villar Palasí (Ley General de Educación de 1970), que, entre otras cosas, impone la Educación General Básica, obligatoria hasta los catorce años. Se supone, por lo tanto, que la población que se incorpora al mercado laboral a partir de 1984 está, en general, educada bajo esta modalidad. Con aquella ley se pretendió racionalizar y homogeneizar el sistema educativo español. Dada la proximidad de esta fecha (1984), no parece posible achacar al cambio educativo los cambios de tendencia en el logro ocupacional que se aprecien entre 1975 y 1995 con suficiente fiabilidad.

\section{EL METODO}

A. Son muchas las diferencias metodológicas entre ambos trabajos. La primera de ellas es muestral. La información del trabajo de 1975 proviene de una muestra de población madrileña cuyo monto ascendía a 430 varones. La comparación de los datos obtenidos con los correspondientes de otras encuestas de alcance nacional (FOESSA, 1967, y el INE, 1968) permite la generalización de los resultados a todo el país con cierta garantía.

La información del trabajo actual, el de 1995, proviene del CIRES. Está formada por la acumulación del bloque de preguntas fijas que acompaña a todas las encuestas realizadas por este centro durante el año 1993. Los entrevistados son mayores de dieciocho años. Por nuestra parte, hemos limitado la población a varones, ocupados y cabezas de familia. La razón de limitar la muestra a los cabeza de familia se encuentra en la sospecha fundada de que la información sobre la renta (renta familiar) de éstos tiene más que ver con las demás variables (ocupación y educación) que en el caso de los demás miembros del hogar. El total de la muestra lo constituyen 3.790 personas.

B. En ambos trabajos se han tenido en cuenta cinco variables, siguiendo la mayoría de los análisis de este tipo:

1. a años de escolaridad del padre;

2. ${ }^{a} \quad$ posición en la escala de prestigio de la ocupación del padre del entrevistado;

3. ${ }^{\text {a }}$ años de escolaridad del entrevistado;

4. ${ }^{a}$ posición en la escala de prestigio de la ocupación del entrevistado;

5. ${ }^{a}$ renta (mes y familia) del entrevistado. 
La definición operativa de las variables es también otra fuente de discrepancia. En general, el criterio de operativización difiere de un trabajo a otro, pese a ciertas semejanzas. Así, en ambos trabajos el nivel educativo se ha contabilizado según el número de años estimado formalmente para adquirir tal nivel. El nivel de renta, a su vez, se mide también en los dos estudios por la marca de clase del respectivo intervalo. Una posible fuente de error por nuestra parte radica en el hecho de que el nivel de renta considerado es el familiar (la suma de todas las fuentes de ingreso familiar), no el individual del padre de familia.

La escala de prestigio ocupacional difiere notablemente de un trabajo a otro. En el de 1975 la escala se construyó a partir de la información de cuatro variables: características de la vivienda; posesión de ciertos bienes de consumo duradero; media de instrucción de los entrevistados, y nivel educativo de los hijos. A partir de la suma de estas variables se obtenía un índice de ordenación de prestigio que se asignaba a la correspondiente categoría ocupacional.

En nuestro caso se ha conseguido una escala de prestigio ocupacional de 0 (mínimo de prestigio) a 100 (máximo), siguiendo criterios ya utilizados por otros autores (D. J. Treiman, 1970: 210), a partir de la media de ingresos y la media educativa de cada una de las 25 categorías ocupacionales que utiliza el CIRES, convenientemente ponderadas. Como posible fuente de error se apunta el haber utilizado la misma escala elaborada para el entrevistado, para clasificar tanto al propio entrevistado cuanto al padre del entrevistado, pese a que seguramente las escalas correspondientes serían algo diferentes.

Ahora bien, pese a tales diferencias, la comparación entre los resultados del modelo de 1975 y el de 1995 es posible debido a los procedimientos del análisis path y a la homogeneidad de las variables (J. Carabaña, 1983: 95).

C. Otra fuente de diferenciación se encuentra en el formato LISREL de nuestro trabajo (sobre los pasos concretos de cálculo, véase Jöreskog y Sörbom/ SPSS Inc., 1989: 119-123). Este formato facilita un conjunto más complejo de relaciones en el modelo de análisis path, tal y como se aprecia en la cantidad de relaciones (flechas) establecidas en el gráfico 2, correspondiente al estudio de 1995, más numerosas que las del gráfico 1, del año 1975. La diferente cantidad de relaciones tiene trascendencia a la hora de establecer la proporción de varianza explicada por el modelo en general, y en la posibilidad de establecer con mayor precisión las relaciones directas e indirectas entre las diferentes variables. Este hecho impide una comparación correcta entre algunos datos del cuadro 4, como en él se indica.

En nuestro caso*, el de 1995, se han considerado dos variables independientes (educación del padre del entrevistado y ocupación del padre del entrevistado) y tres variables dependientes (educación del entrevistado, ocupación

* Agradezco al investigador del Servicio de Informática de la Universidad de Málaga, el doctor don Ramón Hidalgo Sánchez, su colaboración en estos pasos del trabajo. 
del entrevistado y renta del entrevistado). Se ha ideado un modelo recursivo: las variables dependientes pueden ser ordenadas de forma que cada variable dependiente depende tan sólo de las variables independientes y de las dependientes que le preceden. El orden de las variables dependientes es éste: educación del entrevistado, ocupación del entrevistado y renta del entrevistado.

\section{LOS RESULTADOS DE LA COMPARACION}

A. Como ya se indicó, el nivel educativo es el centro de interés de los análisis del logro ocupacional, ya que es la variable, de entre todas las consideradas, sobre la que el sujeto o su familia pueden intervenir más directamente. En efecto, el nivel educativo depende en gran medida de la decisión de seguir estudiando o de abandonar los estudios en algún momento, decisión que a su vez tiene que ver con la definición de la situación (sobre inconvenientes y ventajas del estudio) del estudiante y de su familia.

El origen social, pese a las previsiones de modernización, tiene más que ver con el nivel educativo alcanzado en 1995 que en 1975 (cuadro 2). La proporción de varianza explicada por el origen social en el modelo de J. Díez Nicolás es inferior $(32,4$ por 100$)$ a la nuestra $(37,9)$. Sin embargo, el cambio entre esas dos fechas ha sido complejo: en 1975, la variable que más influía en el nivel educativo alcanzable era la clase ocupacional del padre (coeficiente path 0,34 ); en tanto que la educación del padre tenía menos importancia (coeficiente path $0,24)$. Por contra, la variable que más influye ahora sobre la educación del entrevistado es la educación del padre (coeficiente path 0,49); mientras que la clase ocupacional del padre desciende en importancia (coeficiente path 0,18 ).

Corrobora nuestros datos de 1995 el reciente estudio de J. Rodríguez Menés (1993: 106), en el que el respectivo coeficiente path entre la educación del padre y la del entrevistado es 0,409; mientras que el coeficiente entre la ocupación del padre (cuando el entrevistado tenía dieciséis años) y la educación del entrevistado es mucho menor, 0,137.

B. El acceso a la clase ocupacional por parte del entrevistado ha sufrido también cambios importantes (cuadro 3). En primer lugar, la clase ocupacional de origen pesaba mucho más en la clase ocupacional de pertenencia en el modelo de 1975 (coeficiente path 0,44) que veinte años después (coeficiente path 0,19). Si tenemos en cuenta los efectos totales (los directos más los indirectos) sobre la ocupación del entrevistado, en 1975 la clase ocupacional de origen explicaba el 35,3 por 100 de la varianza de la clase ocupacional de pertenencia; mientras que ese porcentaje queda reducido al 13,5 en 1995.

Además, y esto es seguramente lo más significativo de esta comparación, el nivel educativo se convierte en 1995 en la clave del acceso a la clase ocupacional. El correspondiente coeficiente path entre ambas variables (educación del entrevistado y clase social del entrevistado) es 0,60 , el más alto de todo el 
modelo; en tanto que el respectivo coeficiente en 1975 se quedaba en 0,38 , inferior al coeficiente entre la clase ocupacional de origen y la de pertenencia, también en 1975.

J. Rodríguez Menés presenta parecidos resultados: «... lo que los datos parecen mostrar es una importancia decreciente de la ocupación del padre en la determinación de la primera ocupación del hijo, junto con una tendencia opuesta hacia el reforzamiento de la relación entre los logros educacionales de padres e hijos y las posiciones alcanzadas por estos últimos en la jerarquía ocupacional» (1993: 105).

C. De acuerdo con los datos de 1975 (véase cuado 4), la variable que más tenía que ver con la renta era la ocupación (coeficiente path 0,45), en tanto que el nivel educativo del propio encuestado tenía mucho menos peso directo $(0,13)$. Por el contrario, los datos de 1995 indican unas relaciones causales diferentes. La ocupación ya no es la principal fuente de diferenciación en la renta $(0,27)$, sino la educación $(0,29)$. Estas diferencias se aprecian aún más si comparamos los porcentajes de varianza total explicada. Mientras que en 1975 la educación del entrevistado explicaba el 14,1 por 100 de la varianza de su renta, en 1995 este porcentaje asciende al 24,3. Ocurre al revés con la ocupación del entrevistado: en 1975 explicaba el 26,6 por 100 de la varianza de la renta, y disminuye a un 14 por 100 veinte años más tarde.

Otra diferencia interesante se aprecia en la importancia que tiene el origen social en la renta del entrevistado, mucho mayor en 1975 que en 1995. Así, la varianza total de la renta explicada en 1995 por el origen social alcanza al 18,2 por 100. En 1975, y con un modelo que tiene en cuenta muchos menos enlaces (más incompleto), el porcentaje sube al 24,4 por 100.

D. Se puede resumir todo lo anterior en los siguientes puntos: el nivel educativo se ha ido convirtiendo en el transcurso de estos veinte años en la llave principal para alcanzar la clase ocupacional y como instrumento de reparto de la renta; el origen social influye en la actualidad principalmente, a través de la educación del padre, sobre el nivel educativo y, a través de éste, sobre la ocupación y la distribución de la renta del entrevistado; la influencia directa del origen social sobre la ocupación y la renta es mínima, a diferencia de lo que ocurría en 1975; el nivel educativo del padre tiene ahora más que ver con la carrera ocupacional del entrevistado a través de la educación de éste que con la ocupación del padre; en 1975 era la clase ocupacional del padre, y no su nivel educativo, la que marcaba el futuro educativo y profesional del hijo.

A. H. Halsey presentó unos resultados muy semejantes sobre Gran Bretaña basados en una encuesta realizada en 1972 y cuyo objetivo principal era apreciar el impacto de la Ley de Educación británica de 1944. Entre otros resultados, Halsey afirmaba: el efecto directo de la educación sobre el trabajo está subiendo; el efecto directo de la ocupación del padre sobre el trabajo del entrevistado decae; el efecto directo de la educación del padre sobre el trabajo del entrevistado es casi inexistente (A. H. Halsey, 1975: 182 y 183). 
Para este autor, la educación, cada vez más, sirve de mediador en la transmisión de estatus entre generaciones. Determina el paso de la escuela a la ocupación más fuertemente que en la generación anterior.

\section{MODELO TEORICO DE MODERNIZACION}

El conjunto de relaciones teóricas que se exponen a continuación trata de no salirse de las variables contempladas en los modelos anteriores. Ello nos obliga a prescindir de procesos externos que, sin duda, tienen mucho que ver con los datos anteriores, como, por ejemplo, el aumento de organizaciones formales grandes; el crecimiento del Estado de bienestar; por ende, el aumento de la población ocupada en trabajos no manuales; el desarrollo tecnológico y científico, etc. (J. Burham, 1962; Th. Geiger, 1969; R. Dahrendorf, 1974: 64-70; K. J. Galbraith, 1980; B. Rizzi, 1980; S. Giner y Pérez Yruela, 1972; D. Bell, 1976 y 1977, entre otros).

Además, no se deben olvidar los ciclos económicos de expansión y crisis que contribuyen, a su manera, a desdibujar el proceso de modernización: la expansión favorece, se supone, la individuación, el universalismo; mientras que la crisis impulsa el particularismo y las estrategias colectivas. Aunque no siempre es así. La crisis, por ejemplo, al no crear alternativas laborales a la educación, puede favorecer el exceso de educación formal, con la consecuencia ya apuntada de pérdida del interés económico de la misma (Ch. Freeman, J. Clark y L. Soete, 1985).

A. La idea general implícita en nuestro tipo de análisis, como ya se indicó arriba, reside en la aceptación de una progresiva individuación de la sociedad. En otras palabras, a medida que una sociedad sufre el proceso de modernización, la complejidad social hace que el comportamiento de los individuos sea cada vez menos predecible en función de las categorías sociales de origen y de pertenencia. Este axioma de partida implica, lógicamente, algunas consecuencias esperadas, como, por ejemplo, que cuanto más moderna sea una sociedad, menos tendrá que ver el nivel educativo formal, la clase ocupacional o la renta con el origen social del individuo. Sin embargo, también supone otras consecuencias no tan esperadas: así, la clase ocupacional tendrá que ver cada vez menos con el nivel educativo formal y con la renta; el nivel educativo formal también cada vez menos con la renta, etc. Este proceso de individuación o de independización se encuentra fuertemente ligado al incremento de la productividad y de la renta per capita. Aunque ambos procesos (individuación y productividad) puedan ser clasificados como causa y efecto a la vez, lo que sí parece cierto es que ambos van unidos.

El motor de estos cambios es complejo, pero en gran medida consiste en la presión de los individuos buscando su propio logro ocupacional. El resultado, buscado o imprevisto, es una situación de mayor productividad y eficacia general (S. M. Lipset y H. L. Zetterberg, 1972; R. Boudon, 1978). 
B. A partir del axioma anterior, junto con la información desprendida de los estudios empíricos repasados, se puede establecer el siguiente modelo de cambio social. Como todo proceso de difusión, el proceso de modernización o de individuación no es lineal, sino que su desarrollo se asemeja más a una línea curva en forma de "S», al igual que tantos otros procesos de difusión, social o de cualquier tipo. Tres etapas pueden apreciarse en tal proceso, caracterizadas cada una de ellas por un progresivo aumento de la capacidad de individuación de los actores, así como por otro incremento paralelo de la eficacia productiva del sistema: la primera etapa es aquella en que la distribución de la renta depende de la clase ocupacional de pertenencia, que, a su vez, depende de la clase ocupacional de origen; en la segunda, la distribución de la renta depende en gran medida del nivel educativo alcanzado, que, a su vez, depende también de la educación de origen; la tercera etapa sería aquella otra en la que la distribución de la renta dependiera de otras variables no consideradas en el modelo anterior, tales como habilidades, conocimientos y valías individuales, con independencia de la educación formal conseguida, de la clase ocupacional ocupada y del origen social.

La primera etapa es típica de sociedades en las que no se producen alternativas interesantes, léase rentables, a la reproducción social (es preferible seguir con el negocio de la familia, con la profesión del padre, etc.). La segunda etapa está más abierta a la movilidad y a los determinantes individuales, ya que el nivel educativo depende tanto del origen social cuanto de las habilidades y valías personales. El salto de la primera a la segunda etapa está muy ligado, por un lado, a la obligatoriedad de la enseñanza general y a las facilidades en la oferta educativa (A. H. Halsey, 1977); por otro, a los cambios sustanciales en la estructura ocupacional.

Ahora bien, en esta segunda etapa la individuación de los actores se ve limitada profundamente por la propia naturaleza de la educación formal y del conocimiento. La teoría sobre la utilidad marginal del dinero no sirve precisamente para explicar el interés por adquirir nuevos conocimientos, sino que aquellos que más estudios tienen son los que tienen también más interés en estudiar. O lo que es igual: tiene más interés en leer un libro nuevo el que ha leído muchos libros que el que no ha leído ninguno. Por ello, los padres con niveles altos de educación forzarán a sus hijos para que éstos adquieran también niveles altos educativos, y lo harán con mucho más interés que aquellos cuya educación formal sea escasa.

Así se ha observado en múltiples oportunidades. Se aprecia más interés en invertir en educación por parte de las nuevas clases medias (detentadoras de altos niveles educativos) que en la clase media tradicional o entre los obreros manuales (J. Carabaña, 1993: 227 y 228). De acuerdo con P. Bourdieu, se trata de diferentes estrategias de reproducción social: mientras que las clases medias nuevas utilizan el «capital escolar», la pequeña burguesía utiliza el «capital económico».

Por su parte, R. Boudon explica el proceso afirmando que la clase social inferior calcula los costos de la educación por encima de lo que realmente son, 
en tanto que calcula por debajo de la realidad los beneficios de la misma. Semejante cálculo, aparentemente equivocado, empuja a los muchachos de esta clase social a abandonar los estudios antes que los demás (R. Boudon, 1981: 204).

De cualquier forma, el excesivo interés por la educación, a la vista de su éxito en esta segunda etapa, elevará el nivel de la misma muy por encima de las necesidades productivas de la sociedad. A partir de un exceso de demanda de empleo por parte de una población con altos niveles de educación, decrecerá el interés económico por la educación formal. Es decir, dejará de ser útil como instrumento de selección para ocupar las categorías ocupacionales y, de paso, ya no será causa del desigual reparto de rentas, dando paso a la tercera etapa, en la que continuarán los estudios aquellos cuyo coste general por continuarlos sea menor (más inteligencia, más capacidad de reflexión, más interés, etc.).

A. H. Halsey et al. (1980: 116 y 117), en otro trabajo posterior, denominan a este conjunto de población que tiene menores costes educativos «el pool de habilidades». De acuerdo con estos autores, cada clase social tiene su "nivel de saturación» para cada nivel educativo. A medida que se expande la educación y se hace general (tanto la primaria como la secundaria y, finalmente, la superior), siguiendo un proceso de difusión en " $S$ » ya conocido, se encuentra un tope de capacidad a partir del cual da igual lo bajos que sean los costes de educación, ya que no habrá más individuos capacitados para estudiar que los que ya han estudiado. La tercera etapa teórica señalada por nosotros coincide con la llegada a tal punto de saturación en la clase inferior de la sociedad, clase a la que ese momento de saturación llegará más tarde.

A partir de esta nueva situación, en la que la población haya alcanzado el máximo nivel educativo formal (o nivel de saturación), la eficacia del sistema crecerá seguramente a partir de la conjunción de tres elementos inseparables: la máxima expansión de la educación formal, ya indicada; el reconocimiento de los méritos (habilidades y experiencia) individuales, y, además, por la estrategia socializadora temprana seguida por la familia.

C. Se puede afirmar, a la vista de los resultados de los modelos cotejados en este trabajo, que los datos de 1975 describen una sociedad propia de la primera etapa, en la que prima la clase ocupacional como distribuidora de renta y prestigio; mientras que los de 1995 describen una sociedad en situación intermedia o segunda etapa, en la que el nivel educativo toma el relevo a la clase ocupacional. No se puede olvidar la importancia que ha tenido en el tránsito de la primera a la segunda etapa la Ley de Educación General de 1970, a la vista de que es el acto institucional más importante a la hora de establecer la enseñanza general obligatoria en el país. 


\section{CUADRO 1}

Proporción de varianza explicada según variables (En porcentajes)

\begin{tabular}{|c|c|c|}
\hline Variables dependientes & 1975 & 1995 \\
\hline 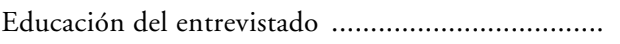 & 28 & 62 \\
\hline Ocupación del entrevistado ...................................... & 50 & 72 \\
\hline 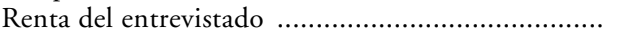 & 41 & 58 \\
\hline
\end{tabular}

FuENTES: J. Díez Nicolás et al. (1975) y CIRES (1993). Elaboración propia.

\section{CUADRO 2}

Influencias del origen social sobre la educación del entrevistado

\begin{tabular}{|c|c|c|}
\hline & 1975 & 1995 \\
\hline & \multicolumn{2}{|c|}{ Efectos directos sobre la educación } \\
\hline Educación del padre & 0,24 & 0,49 \\
\hline \multirow{2}{*}{ 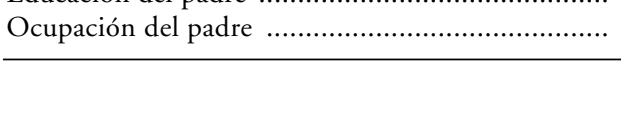 } & 0,44 & 0,18 \\
\hline & \multicolumn{2}{|c|}{ Varianza total explicada } \\
\hline 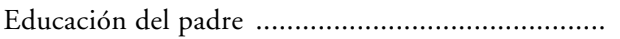 & $10,8 \%$ & $29,4 \%$ \\
\hline 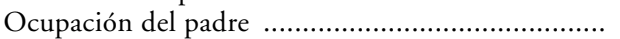 & $21,6 \%$ & $8,5 \%$ \\
\hline \multirow[t]{2}{*}{ 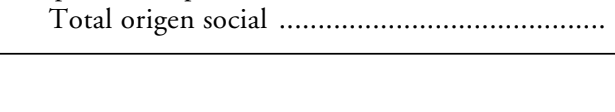 } & $32,4 \%$ & $37,9 \%$ \\
\hline & \multicolumn{2}{|c|}{ Valores de $T^{*}$} \\
\hline 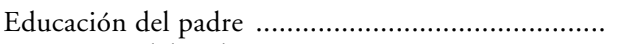 & - & 31,3 \\
\hline 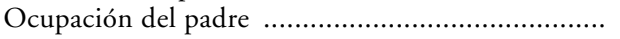 & - & 11,7 \\
\hline
\end{tabular}

* Influencia significativa cuando $\mathrm{T}>1,96$.

Fuentes: J. Díez Nicolás et al. (1975) y CIRES (1993). Elaboración propia. 


\section{CUADRO 3}

Influencias del origen social y de la educación sobre la ocupación del entrevistado

\begin{tabular}{|c|c|c|}
\hline & 1975 & 1995 \\
\hline & \multicolumn{2}{|c|}{ Efectos directos sobre la ocupación } \\
\hline 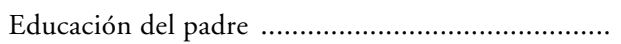 & - & $-0,04$ \\
\hline 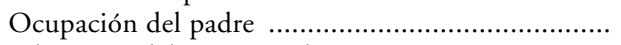 & 0,44 & 0,19 \\
\hline 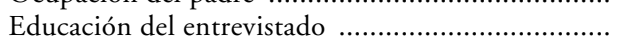 & 0,38 & 0,60 \\
\hline
\end{tabular}

Efectos indirectos sobre la ocupación

Educación del padre

0,09

0,29

Ocupación del padre

0,13

0,11

Efectos totales sobre la ocupación

Educación del padre

- $\quad 0,26$

Ocupación del padre

0,57

0,30

Educación del entrevistado

0,38

0,60

Varianza total explicada

Educación del padre

- $\quad 11,2 \%$

Ocupación del padre

$35,3 \%$

$13,5 \%$

Total origen social

-

$24,7 \%$

Educación del entrevistado

$22,4 \%$

$40,2 \%$

Valores de $T^{*}$

Educación del padre

$\begin{array}{ll}- & -2,4 \\ - & 12,9 \\ - & 39,9\end{array}$

Ocupación del padre 39,9

* Influencia significativa cuando $\mathrm{T}>1,96$.

FUENTES: J. Díez Nicolás et al. (1975) y CIRES (1993). Elaboración propia. 


\section{CUADRO 4}

Influencias del origen social, la educación y la ocupación sobre la renta

\begin{tabular}{|c|c|c|}
\hline & 1975 & 1995 \\
\hline & \multicolumn{2}{|c|}{ Efectos directos sobre la renta } \\
\hline Educación del padre & 0,17 & 0,05 \\
\hline 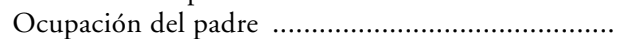 & - & 0,08 \\
\hline 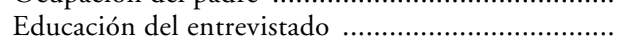 & 0,13 & 0,29 \\
\hline 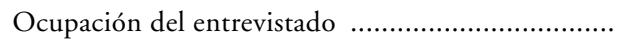 & 0,45 & 0,27 \\
\hline
\end{tabular}

Efectos indirectos sobre la renta

Educación del padre

$0,07^{* *} \quad 0,21$

Ocupación del padre

0,30

0,14

Educación del entrevistado

0,17

0,17

Efectos totales sobre la renta

Educación del padre

$\begin{array}{ll}0,24^{* *} & 0,26 \\ 0,30^{* *} & 0,22 \\ 0,30 & 0,45 \\ 0,45 & 0,27\end{array}$

Varianza total explicada

Educación del padre

$\begin{array}{lr}10,3 \%^{* *} & 10,1 \% \\ 14,1 \%^{* *} & 8,1 \% \\ 14,1 \% & 24,3 \% \\ 26,6 \% & 14,0 \%\end{array}$

Ocupación del padre

Educación del entrevistado

$26,6 \%$

Valores de $T^{*}$

Educación del padre

$\begin{array}{rr}- & 2,7 \\ - & 4,9 \\ - & 14,6 \\ - & 15,3\end{array}$

Ocupación del padre

Educación del entrevistado

15,3

Ocupación del entrevistado

* Influencia significativa cuando $\mathrm{T}>1,96$.

** Datos no comparables.

FUENTES: J. Díez Nicolás et al. (1975) y CIRES (1993). Elaboración propia. 


\section{GRAFICO 1}

Análisis path de la influencia del origen social sobre la educación, la ocupación y la renta (1975)

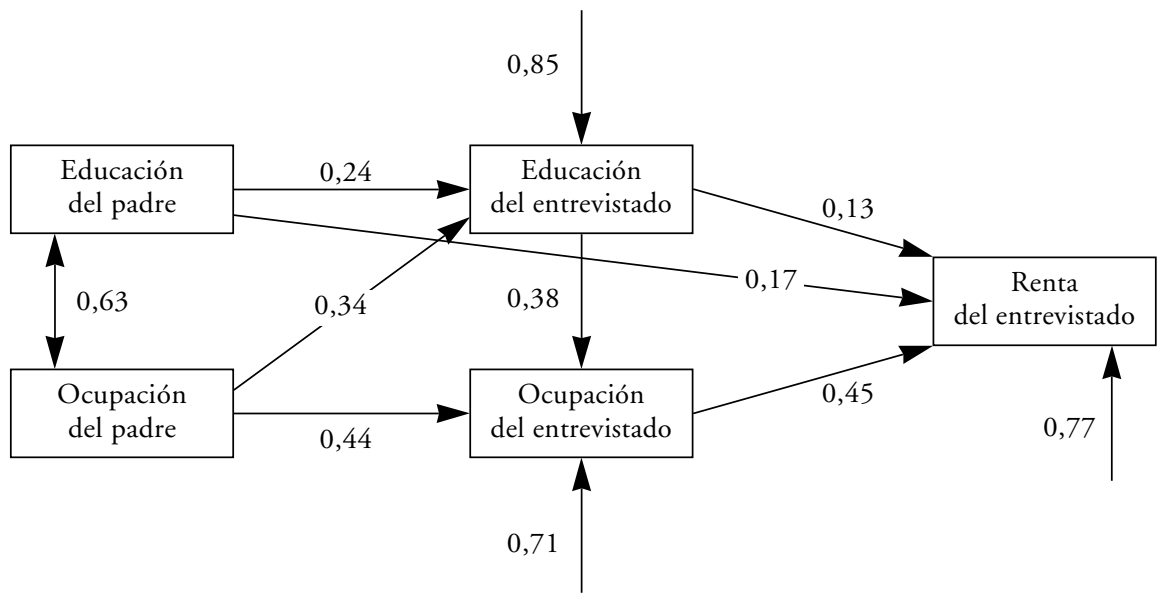

Fuente: J. Díez Nicolás et al. (1975).

\section{GRAFICO 2}

Análisis path de la influencia del origen social sobre la educación, la ocupación y la renta (1995)

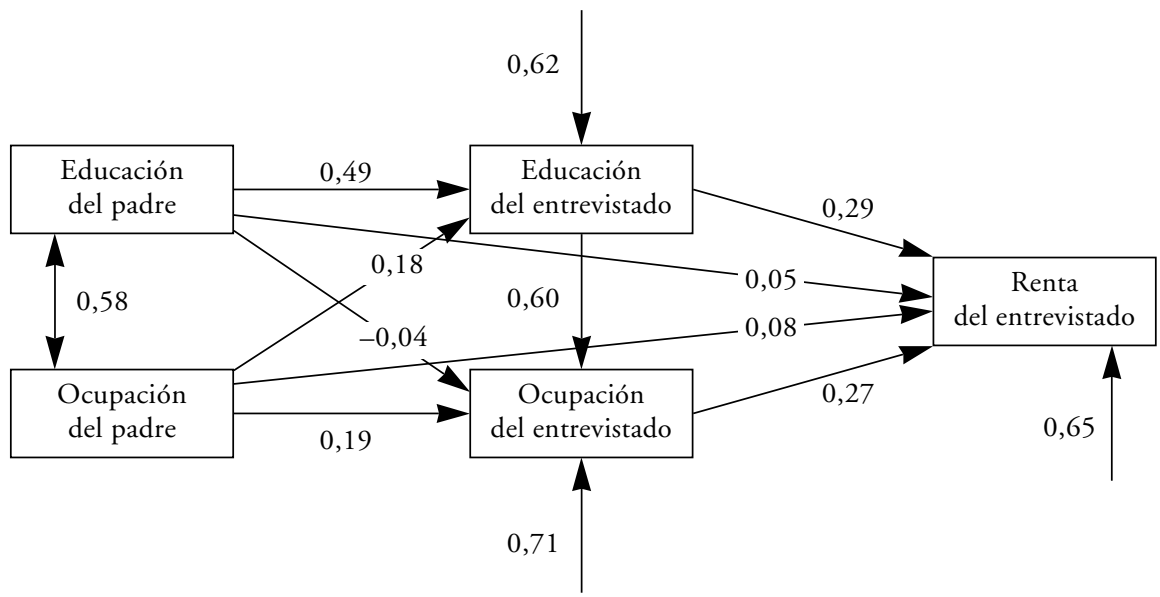

FUENTE: CIRES (1993). Elaboración propia. 


\section{BIBLIOGRAFIA}

BELL, D. (1976): El advenimiento de la sociedad post-industrial, Alianza, Madrid.

- (1977): Las contradicciones culturales del capitalismo (1. a ed., 1976), Alianza, Madrid.

Blau, P. M., y Duncan, O. D. (1967): The American Occupational Structure, Free Press, Nueva York.

Boudon, R. (1979): Effets pervers et ordre social, PUF, París.

- (1981): La lógica de lo social, Rialp, Madrid.

- (1983): La desigualdad de oportunidades, Laia, Barcelona.

Burnham, J. (1962): La revolución de los directores, Huemul, Buenos Aires.

CACHÓN RODRÍGUEZ, L. (1989): ¿Movilidad social o trayectorias de clase?, CIS, Madrid.

CARABAÑA, J. (1983): Educación, ocupación e ingresos en la España del siglo XX, Ministerio de Educación y Ciencia, Madrid.

- (1993): «Sistemas de enseñanza y clases sociales», en García de León et al. (eds.), Sociología de la educación, Barcanova, Barcelona, pp. 207-252.

Coser, L. A. (1975): «Presidential Address: Two Methods in Search of a Substance», American Sociological Review, vol. 40, núm. 6, pp. 691-700.

DAHRENDORF, R. (1974): Las clases sociales y su conflicto en la sociedad industrial (1. a ed., 1957), Rialp, Madrid.

Díez Nicolás, J.; Martínez lázaro, U., y Porro Minondo, M. J. (1975): «Education and Social Mobility in Spain», en OCDE, Education, Inequality and Life Chances, París, pp. 563612.

Freeman, Ch.; Clark, J., y Soete, L. (1985): Desempleo e innovación tecnológica (1.a ed., 1982), Ministerio de Trabajo y Seguridad Social, Madrid.

Galbraith, K. J. (1980): El nuevo estado industrial (1.a ed., 1968), Ariel, Barcelona.

Geiger, Th. (1969): "Class Society in the Melting Pot» (1949), en C. S. Heller (comp.), Structured Social Inequality, The McMillan Company, Nueva York, pp. 91-104.

Giner, S., y PÉrez Yruela, M. (1972): La sociedad corporativa, CIS, Madrid.

GIROD, R. (1977): Inegalité, inegalités, PUF, París.

Halsey, A. H. (1977): «Towards Meritocracy? The Case of Britain», en J. Karabel y A. H. Halsey, Power and Ideology in Education, Oxford University Press, Oxford, pp. 173-185.

Halsey, A. H.; Heath, A. F., y Ridge, J. M. (1980): Origins and Destinations. Family, Class and Education in Modern Britain, Clarendon Press, Oxford.

HERNÁNDEZ DE FrUTOS, T. (1993): «El status attainment a mitad de camino entre teoría y técnica analítica", Revista Española de Investigaciones Sociológicas, núm. 61, pp. 185-198.

JÖresKog y SÖRbOM/SPSS (1989): LISREL 7. A Guide to the Program and Applications. SPSS Statistical Path Analysis, Chicago.

Knottnerus, J. D. (1987): «Status Attainment research and its image of Society», American Sociological Review, vol. 52, núm. 1, pp. 113-121.

Krymkowski, D. H. (1991): "The Process of Status Attainment among Men in Poland, the US, and West Germany», American Sociological Review, vol. 56, núm. 1, pp. 46-59.

Requena Santos, F. (1991): «Social Resources and Occupational Status Attainment in Spain: A Cross-National Comparison with the United States and Netherlands", International Journal of Comparative Sociology, XXXII, 3-4, pp. 233-242.

Rizzi, B. (1980): La burocratización del mundo, Península, Barcelona.

Rodríguez MEnés, J. (1993): «Movilidad social y cambio social en España», Revista Española de Investigaciones Sociológicas, núm. 61, pp. 77-125.

Rossides, D. W. (1990): Social Stratification. The American Class System in Comparative Perspective, Prentice Hall, Englewood Cliffs, Nueva Jersey.

Thurow, L. C. (1977): «Education and Economic Equality», en J. Karabel y A. H. Halsey, Power and Ideology in Education, Oxford University Press, Oxford, pp. 325-334.

Treiman, D. J. (1970): «Industrialization and Social Stratification», en E. O. Laumann (ed.), Social Stratification: Research and Theory for the 1970s, The Bobbs-Merril Company, Inc., Nueva York, pp. 207-234. 


\title{
RESUMEN
}

Este trabajo es una propuesta de modelo de cambio social que toma como variable más significativa el grado de expansión de la educación formal. Supone tal modelo la existencia de tres etapas, según el interés que despierte la educación formal como instrumento de movilidad social. En una primera etapa, la educación apenas tiene importancia para la mejora de fortuna; en la segunda, es ciertamente importante; en la tercera etapa, se supone que perderá de nuevo todo su interés al respecto. La verificación del modelo se apoya en la comparación de los resultados del primer análisis de camino efectuado sobre población española en 1975, que responde a los criterios de la primera etapa del modelo, y otro realizado en la actualidad siguiendo parecida metodología, que describe una sociedad propia de la segunda etapa.

\begin{abstract}
In this paper we put forward a model of social change whose most important variable is the degree of spreading of formal education. The above mentioned model involves three stages, according to the interest aroused by formal education, considered as an instrument of social mobility. In the first stage, education is hardly important as means of economic improvement; in the second stage, it certainly becomes important; in the third stage, education is believed to have little importance again. The model has been checked by comparing the results of an path analysis carried out in Spain in 1975 (first stage) with another one, carried out nowadays with a similar method, which describes a society in the second stage of the model.
\end{abstract}

